\title{
Ortopedia funcional dos maxilares, respiração bucal e distúrbios respiratórios do sono em crianças
}

\author{
Functional orthopedic, mouth breathing, and sleep respiratory \\ disorder in children.
}

Débora Aparecida Lentini-Oliveira ${ }^{1}$, Fernando Rodrigues Carvalho², Marco Antônio Cardoso Machado ${ }^{3}$, Lucila Bizari Fernandes Prado ${ }^{4}$, Gilmar Fernandes do Prado ${ }^{5}$

\begin{abstract}
RESUMO
A relação entre morfologia facial, respiração bucal e distúrbios respiratórios do sono vem sendo discutida, mas ainda não tem suporte científico. Os objetivos desse artigo são: fazer uma atualização do conhecimento sobre a relação entre respiração bucal, distúrbios respiratórios do sono e determinadas más-oclusões; enfatizar a importância da interdisciplinaridade na busca de melhor prognóstico e estabilidade de tratamento, tanto na correção dos distúrbios respiratórios do sono como na correção das más-oclusões.
\end{abstract}

Unitermos: Maloclusão, Respiração bucal, Ronco, Apnéia do sono tipo obstrutiva .

Citação: Lentini-Oliveira DA, Carvalho FR, Machado MAC, Prado LBF, Prado GF. Ortopedia funcional dos maxilares, respiração bucal e distúrbios respiratórios do sono em crianças. Rev Neurociencias 2005; 13(2): 087-092.

\section{SUMMARY}

The relationship between facial morphology oral breathing and sleep breathing disorder has been discussed, but the relation does not have been supported scientifically yet. The article's objectives are: actualize the knowledge about the relationship between oral breathing, sleep breathing disorder and several malocclusions; to emphasize the importance of the treatment among the several disciplines looking for the best prognostic and treatment stability, so much in the correction of the disturbances of the sleep as in the relationship of the malocclusion

Keywords: Malocclusion, Mouth breathing, Snoring, Obstructive sleep apnea.

Citation: Lentini-Oliveira DA, Carvalho FR, Machado MAC, Prado LBF, Prado GF. Functional orthopedic, mouth breathing, and sleep respiratory disorder in children. Rev Neurociencias 2005; 13(2): 087-092.

\footnotetext{
Trabalho realizado: Universidade Federal de São Paulo, UNIFESP, São Paulo, SP.

1 - Especilista em odontopediatria, Faculdade Odontologia de Bauru - USP, Pós-graduando em Medicina Interna e Terapêutica, Universidade Federal de São Paulo, UNIFESP, São Paulo, SP.

2 - Especialista em Ortopedia Funcional dos Maxilares, Professor da Sociedade Paulista de Ortodontia e Ortopedia Funcional dos Maxilares, Pós-graduando em Medicina Interna e Terapêutica, Universidade Federal de São Paulo, UNIFESP, São Paulo, SP.

3 - Pós-graduando em Medicina Interna e Terapêutica, Universidade Federal de São Paulo, UNIFESP, São Paulo, SP.

4 - Diretora do Laboratório Neuro-Sono da Disciplina de Neurologia, Universidade Federal de São Paulo, UNIFESP, São Paulo, SP.

5 - Professor Adjunto da Disciplina de Medicina de Urgência, Universidade Federal de São Paulo, UNIFESP, São Paulo, SP.
}

Endereço para correspondência: Débora Aparecida Lentini-Oliveira

Rua Marechal Dutra, 691

Jardim Mariana, Sorocaba - SP

CEP: $18040-792$

E-mail: deblentiniaterra.com.br 


\section{INTRODUÇÃO}

Os distúrbios respiratórios do sono, em crianças, têm sido reconhecidos como um processo patológico importante e contínuo, que pode incluir: ronco, obstrução das vias aéreas superiores e eventos apnêicos. A prevalência tem sido estimada em 12,1\% ${ }^{1}$ e o pico de incidência ocorre entre 3-6 anos de idade ${ }^{2}$.

Embora a demora no diagnóstico e tratamento possam provocar comprometimento físico e comportamental como: déficit de atenção, hiperatividade déficit cognitivo $^{1-5}$, baixa estatura ${ }^{2,6}$ hipertensão pulmonar e cor pulmonale ${ }^{2}$, recentes evidências indicam que os sintomas são variados e difíceis de detectar. Há falta de consenso nas definições, nos critérios diagnósticos e muitas questões sem respostas ${ }^{7}$.

Por outro lado, a relação entre respiração bucal e morfologia facial também tem sido discutida na literatura.

Embora estudos considerem a influência da respiração bucal nas alterações da morfologia facial, como acentuação do padrão vertical no crescimento facial, mordidas abertas e mordidas cruzadas ${ }^{8-11}$, dados de um estudo longitudinal indicam que os efeitos do modo respiratório na morfologia facial são insuportados ${ }^{12}$. Esta relação de causa e efeito não é totalmente conhecida.

Avaliações ortodônticas em crianças com apnéia obstrutiva do sono encontram características semelhantes: mordidas cruzadas unilaterais, mordidas abertas e incompetência labial ${ }^{13}$.

O tratamento de escolha da apnéia obstrutiva do sono, em crianças, têm sido a adenotonsilectomia ${ }^{14}$.

Embora estudos reportem significante melhora após estas cirurgias ${ }^{15,16}$, outros estudos têm demonstrado desordens respiratórias residuais em polissonografia nessas crianças ${ }^{17,18}$.

Essas crianças que não melhoram após cirurgia apresentam estreitamento do espaço aéreo epifaríngeo, desenvolvimento maxilar deficiente e retrusão mandibular ${ }^{15}$. Diversos tratamentos ortopédicos funcionais dos maxilares ou ortodônticos têm sido propostos para correção das diferentes másoclusões. Embora a avaliação da respiração bucal seja rotineira na clínica ortopédica e ortodôntica, pouca atenção tem sido dispensada por esses profissionais aos distúrbios respiratórios do sono. Os objetivos desse estudo são:

1 - Atualização sobre a relação entre respiração bucal, distúrbios respiratórios do sono, e determinadas más-oclusões;
2 - Enfatizar a importância da interdisciplinaridade na busca de melhor prognóstico e estabilidade de tratamento, tanto na correção dos distúrbios respiratórios do sono como na correção das másoclusões.

\section{SÍNDROME DA APNÉIA OBSTRUTIVA DO SONO (SAOS) EM CRIANÇAS}

É um distúrbio respiratório que acontece durante o sono caracterizado por obstrução parcial ou total da ventilação associada com dessaturação de oxigênio, hipercapnia e sintomas que incluem ronco habitual, dificuldade respiratória e problemas comportamentais diurnos, tais como déficit de atenção, hiperatividade e déficit cognitivo. As complicações podem incluir: déficit pôndero-estatural, desordens neurológicas e cor pulmonale ${ }^{19}$.

Alguns fatores têm sido considerados como possíveis fatores etiológicos: hipertrofia de amígdalas e de adenóides, predisposição familiar à hipoxemia, hipercapnia ou a obesidade e ainda a determinadas características faciais e crâniomandibulares.

Estudo experimental ${ }^{8}$ em macacos mostrou a relação entre obstrução nasal forçada e modificação no padrão de crescimento facial, com retorno à condição anterior após a desobstrução.

Embora a respiração bucal esteja freqüentemente associada com deformidades dentofaciais, há registros de respiração bucal tanto em más-oclusões como em oclusões normais. Doenças inflamatórias das vias aéreas como asma e rinites alérgicas, que levam à respiração bucal, têm uma forte, mas pouco entendida relação tanto com anormalidades faciais como com distúrbios respiratórios do sono ${ }^{20}$.

Para o diagnóstico da SAOS, a polissonografia (PSG) é reconhecida como exame padrão ouro. Em crianças, o registro de um único evento de apnéia por hora já caracteriza a síndrome da apnéia obstrutiva do sono ${ }^{21}$.

Nem sempre a avaliação clínica sugestiva de apnéia apresenta resultado polissonográfico positivo para apnéia, mas pode apontar características de anormalidade, tais como esforço respiratório anormal, medido diretamente pela pressão esofágica ou acompanhado por microdespertares ou despertares indicado pelo eletroencefalograma. Assim os distúrbios respiratórios do sono incluem a Síndrome da apnéia obstrutiva e a Síndrome do Aumento de Resistência das Vias Aéreas Superiores ${ }^{22}$. 
As opções de tratamento incluem: descongestionantes nasais, esteróides intranasais, perda de peso em crianças obesas, pressão aérea positiva contínua (CPAP), pressão aérea positiva em dois níveis (BIPAP) e, principalmente, adenotonsilectomia em crianças com hipertrofia adenotonsilar ${ }^{7}$.

Embora os critérios diagnósticos não sejam bem definidos, os principais fatores etiológicos considerados são hipertrofia de amígdalas e adenóides.

Portanto, o tratamento de escolha para apnéia obstrutiva do sono em crianças tem sido a adenotonsilectomia ${ }^{23}$.

A adenotonsilectomia produz resultados tanto na apnéia obstrutiva do sono ${ }^{14-16}$, como no padrão de crescimento facial, com melhora na largura dos arcos dentais e na inclinação dos dentes incisivos ${ }^{10}$.

Em função do tempo de persistência da respiração bucal e da magnitude da alteração de maxila, mandíbula e das inclinações dentárias, junto com características hereditárias, há possibilidade de persistência de alterações oclusais que pode contribuir para persistência dos sintomas da apnéia.

Uma revisão sistemática da literatura realizada junto ao Instituto Cochrane ${ }^{23}$ sobre o efeito da adenotonsilectomia em apnéia obstrutiva do sono em crianças foi inconclusiva em função de problemas metodológicos nos estudos, que incluía falta de randomização e controles e variações nas definições de variáveis chaves.

\section{MÁS-OCLUSÕES E ORTOPEDIA FUNCIO- NAL DOS MAXILARES}

\section{Más-oclusões}

O crescimento dos ossos da face ocorre em diferentes direções, velocidades e quantidades a partir de sinais precisos e coordenados enviados aos seus tecidos conjuntivos. Esses sinais podem ser hormônios, potenciais bioelétricos e forças mecânicas, que agem como ativadores extracelulares a que receptores específicos das superfícies celulares são sensíveis. Essa recepção desencadeia uma cascata de mensageiros secundários num determinado tipo de célula, ativando a síntese de enzimas relacionadas com deposição ou reabsorção óssea.

Os eventos de crescimento e desenvolvimento maxilo-mandibular não são totalmente conhecidos, mas há uma tentativa de busca de equilíbrio estrutural e funcional, entre tecidos moles e tecidos du- ros. Múltiplos fatores são envolvidos no controle do crescimento maxilo-mandibular, e a faixa de desequilíbrio é estreita ${ }^{24}$.

Todas as más-oclusões freqüentemente são resultado da combinação de diferentes fatores dentro de um potencial de crescimento inerente a cada indivíduo e, portanto, são multifatoriais.

As más-oclusões que têm sido associadas com respiração bucal em crianças são descritas a seguir:

\subsection{Mordida cruzada}

A mordida cruzada lateral funcional caracteriza-se por atresia maxilar, instabilidade oclusal e, portanto, desvio de posição mandibular para um dos lados para possibilitar a função mastigatória, estabelecendo-se o cruzamento da mordida ${ }^{25}$.

Há uma relação entre a largura do arco superior, respiração bucal e posição de língua baixa associada com adenóide ${ }^{10}$.

Moss, na sua teoria da matriz funcional, enfatiza o papel da respiração nasal no desenvolvimento maxilar e abaixamento da abóbada palatina ${ }^{26}$. Quando ocorrem obstruções à respiração nasal, a maxila fica atrésica.

Também pode ocorrer mordida cruzada anterior funcional ou estrutural em função de discrepâncias sagitais, seja por deficiência de desenvolvimento maxilar ou excesso de desenvolvimento mandibular, as chamadas mésiooclusões ou classe III de Angle.

\subsection{Mordida aberta anterior}

É definida como negativa sobremordida vertical entre dentes antagonistas anteriores ou uma falta de contatos, em direção vertical, entre segmentos opostos de dentes ${ }^{27}$. A etiologia da mordida aberta anterior é discutida e os fatores associados são os fatores predisponentes associados a outros, como hábitos não nutritivos, função anormal da língua, hipertrofia de amígdalas e adenóides que levariam a uma respiração buca|28. A mordida aberta esquelética caracterizase por incompetência labial e características cefalométricas comuns às encontradas em indivíduos com apnéia obstrutiva do sono $^{29}$ e respiradores bucais ${ }^{30}$ : face longa e aumento da altura facial anterior inferior (distância queixo-nariz). Um estudo comparou forças oclusais entre crianças e adultos normais e com face longa e os resulta- 
dos sugerem que indivíduos com esse padrão, falham em ganhar força nos músculos elevadores da mandíbula ${ }^{31}$. Também nesse estudo, os indivíduos com face longa eram mais retrognatas.

\subsection{Retrognatismo}

O retrognatismo, comumente encontrado nos indivíduos classe II de Angle, caracteriza-se por um relacionamento distal da mandíbula em relação à maxila. A língua fica posteriorizada, tornando-se um importante fator de obstrução à passagem de ar durante o sono nessa região retro-lingual.

Em adultos, essa relação com apnéia obstrutiva do sono já é conhecida e dependendo da severidade da apnéia, aparelhos reposicionadores intra-orais são indicados.

Essas más-oclusões parecem ter uma provável relação com distúrbios respiratórios do sono.

\section{Ortopedia Funcional dos Maxilares}

A Ortopedia Funcional dos Maxilares é uma especialidade da Odontologia que tem por objetivo monitorar o desenvolvimento da oclusão, eliminando os impedimentos à harmonia de desenvolvimento e corrigindo os desvios da função oclusal, através de recursos próprios que podem ser aparelhos em dentes decíduos ou permanentes, ajustes oclusais por desgastes ou ajustes oclusais por acréscimo de resinas em dentes decíduos.

Também visa o equilíbrio estético e normalização das funções: mastigação, deglutição, respiração e fonação.

A Ortopedia Funcional dos Maxilares atua redimensionando o crescimento e desenvolvimento maxilo-mandibular e face, através de estímulos aplicados com direção e velocidade adequados ${ }^{32}$.

De uma maneira simplificada, os princípios de ação das técnicas ortopédicas funcionais incluem mudanças de tônus muscular na face, através de uma mudança na relação dinâmica da mandíbula em relação à maxila e mudança na postura de língua. Essas mudanças de posição e movimento são captadas pelos proprioceptores (fusos neuro-musculares, órgãos tendinosos, receptores articulares). Essa percepção é conduzida até o sistema nervoso central e a resposta inclui novas mudanças de tônus muscular, portanto, ativação e desativação dos mecanismos de deposição e reabsorção óssea ou remodelação.
O objetivo final é que a maxila e mandíbula estejam com seus perímetros compatíveis e com condições de contatos oclusais simultâneos e simétricos e dimensões verticais iguais em ambos os lados da boca. Os movimentos mandibulares, durante as funções devem ser livres, sem mudança de direção. Essas características permitem que uma mastigação bilateral e alternada ocorra dentro de condições de equilíbrio neuromuscular e, portanto, a harmonia de desenvolvimento pode se manter ${ }^{33}$.

Rinopatias ou hipertrofias de adenóides e amígdalas podem prejudicar ou impedir o sucesso dos tratamentos.

\section{Diagnóstico em Ortopedia Funcional dos Maxilares}

O diagnóstico em Ortopedia Funcional dos Maxilares inclui anamnese, análise facial, análise estática das relações maxilo-mandibulares, análise estática da oclusão e análise da dinâmica mandibular ou análise funcional da oclusão. A base do diagnóstico é o exame clínico da criança complementado por exames radiográficos, estudos cefalométricos, análise de modelos que relacionam o plano oclusal com planos faciais: plano de Camper, plano sagital mediano e plano frontal. Alguns aspectos do diagnóstico clínico, especificamente da análise facial e análise estática da oclusão podem auxiliar o neurologista, especialista em sono, na observação de alterações que podem estar relacionadas.

\subsection{Análise facial}

Com ela, avalia-se a simetria facial: as proporções faciais nos terços superior, médio e inferior da face em vista frontal e de perfil , com o paciente em posição natural de cabeça. Deve existir uma relação de proporcionalidade entre os terços facial superior (linha do cabelo à sombrancelha), médio (sombrancelha ao ponto subnasal) e inferior (ponto subnasal ao tecido mole do mente)

Avalia-se também a simetria entre os lados direito e esquerdo do indivíduo (norma frontal) tanto em largura como em altura.

Deve-se também observar se há selamento labial, tonicidade de lábios e mento. Um bom selamento labial sugere ausências de discrepâncias esqueléticas verticais e sagitais, comprimento labiais adequados e altura facial inferior proporcional aos tamanhos de maxila e mandíbula, função respiratória normal e tonicidade labial normal. 
Indivíduos com retrognatismo podem apresentar ausência de selamento labial, interposição labial inferior entre os dentes, selamento labial forçado com hipertrofia de contração do músculo mentoneano. Em norma lateral, apresentam perfil convexo e comprimento de linha queixo-pescoço pequena. Ao contrário, as mésio-oclusões podem apresentar linha queixo-pescoço longa e ângulo queixo-pescoço agudo. Esse ângulo agudo é indicativo de padrão de crescimento facial mais vertical, característico também da mordida aberta estrutural, respiradores bucais e dos adultos apneicos.

As deficiências de crescimento maxilar podem ser observadas em norma lateral , por face côncava ou deficiência na região zigomática.

\section{CONSIDERAÇÕES FINAIS}

A hipótese de que a alteração do modo respiratório pode alterar tanto crescimento da maxila, que permanece atrésica, como da mandíbula que sofre rotação de crescimento e postura da língua, modificando o padrão de crescimento facial e tonicidade muscular da face, deve ser ainda bastante pesquisa- da. Assim como, essas relações com os distúrbios respiratórios do sono.

É fundamental que os profissionais médicos que lidam com os distúrbios respiratórios do sono em crianças estejam atentos também às características faciais e maxilo-mandibulares que possam estar contribuindo com os processos de obstrução ou que possam impedir a recuperação total do indivíduo.

Ao mesmo tempo, os ortopedistas e ortodontistas, que tratam as más-oclusões, também precisam incluir no seu diagnóstico, a observação cuidadosa de sinais e sintomas que possam sugerir distúrbios respiratórios do sono, para encaminhar devidamente esses pacientes, melhorando não só o seu prognóstico, mas principalmente evitando os danos que esses distúrbios podem provocar.

Em função da complexidade, falta de consenso entre critérios diagnósticos e da importância clínica dos distúrbios respiratórios do sono em crianças, todos os fatores associados devem ser diagnosticados e tratados por equipe multidisciplinar, dentro de ensaios clínicos controlados e randomizados e avaliados para a possível melhora do prognóstico a longo prazo.

\section{REFERÊNCIAS BIBLIOGRÁFICAS}

1. Ali NJ, Pistson DJ. Snoring, sleep disturbance, and behaviour in 4-5 years old. Arch Dis Child 1993; 68: 360-366.

2. Brouillette RT, Fernbach SK, Hunt CE. Obstructive sleep apnea in infants and children. J Pediatr 1982; 100:31-40.

3. O'Brien LM, Tauman R, Gozal D. Sleep pressure correlates of cognitive and behavioral morbidity in snoring children. Sleep 2004; 27:279-282.

4. Gottlieb DJ, Vezina RM, Chase C et al. Symptoms of sleep-disordered breathing in 5-year-old children are associated with sleepiness and problem behaviors. Pediatrics 2003; $112(4)$ 870-877.

5. Guilleminaut C, Winkle R, Korobkin R, Simmons B. Children and nocturnal snoring: evaluation of the effects of sleep related respiratory resistive load and daytime functioning. Eur J Pediatr 1982; 139:165-171.
6. Greene MG. Consequences of sleep disordered breathing in children. Curr Ipen Pulm Med 1997; 3: 456-63.

7. Carrol JL Obstructive sleep-disordered breathing in children: new controversies, new directions. Clin Chest Med 2003; 24(2) 261-282.

8. Harvold EP. Experiments on development of dental malocclusion. Am J Orthod 1972; 61: 38-44.

9. Linder-Aronson S. Adenoids - Their effect on mode of breathing and nasal airflow and their relationship to characteristics of the facial skeleton and the dentition. Acta Otolaryngol 1970; 265(suppl): 1-132.

10. Linder-Aronson S. Effects of adenoidectomy on dentition and nasopharynx. Am J Orthod 1974; 65:1-15.

11. Ricketts R. Respiratory obstruction syndrome. Am J Orthod 1968;54 (7): 495-507. 
12. Shanker S, Fields HW, Beck FM, Vig PS, Vig KWL. A longitudinal assessment of upper respiratory function and dentofacial morphology in 8-12-year-old children. Seminars in Orthodontics 2004; 10(1):45-53.

13. Caprioglio A, Zucconi M, Calori G, Troiani V. Habitual snoring OSA and craniofacial modification. Orthodontic clinical and diagnostic aspects in a case control study. Minerva Stomatol 1999; 48(4):125-137.

14. Helfaer MA, MacColley SA, Pyzik PL et al. Polysomnography after adenotonsillectomy in mild pediatric obstructive sleep apnea. Crit Care Med 1996; 24(8): 1-10.

15. Shintani T, Asakura K, Kataura A. The effect of adenotonsillectomy in children with OSA. Int J Pediatr Otorhinolaryngol 1998; 44:51-58.

16. Goldestein NA, Pugazhendhi V, Rao SM et al. Clinical assessment of pediatric obstructive sleep apnea. Pediatrics 2004; 114(1): 33-43.

17. Guilleminault C, Biol D, LiK, Quo S, Randall N. A prospective study on the surgical outcomes of children with sleepdisordered breathing. Sleep 2004; 27(1): 95-100.

18. Tasker C, Crosby JH, Stradeling JR. Evidence for persistence of upper airway narrow during sleep 12 years after adenotonsillectomy. Arch Dis Child 2002; 86:34-37.

19. Schechter MS. Section on Pediatr Pulmonology, Subcommittee on Obstructive Sleep Apnea Sindrome. Technical report: diagnosis and management of childhood obstructive sleep apnea syndrome. Pediatrics 2002; 109(4): 1-20.

20. Preston CB. Seminars in Orthodontics - Introduction. Seminars in Orthodontics 2004, 10(1): 1-2.

21. Uliel S, Tauman R, Greenfeld M, Sivan Y. Normal Polysomnographic Respiratory Values in Children and Adolescents.
Chest 2004; 125(3): 872-878.

22. Guilleminault $\mathrm{C}$, Pelayo R. Sleep-disordered breathing in children. Ann Med 1998; 30(4): 350-356.

23. Lim J, Mckean M Adenotonsillectomy for obstructive sleep apnoea in children. In: The Cochrane Library, Issue 4, 2004.

24. Enlow DH, Poston WR, Bakor SF. Crescimento facial. $3^{\mathrm{a} e d i-}$ ção. Porto Alegre: Artes Médicas, 1993.

25. Silva Filho OG, Pinto DM, Alvares LC. Alterações condilares associadas às mordidas cruzadas funcionais. Ortodontia, 1997; 25(2): 41-51.

26. Moss ML. The functional matrix. In: Kraus BS, Riedel RA. Vistas in Orthodontics. Philadelphia: Lea\& Febiger Inc, 1962.

27. Subtelny JE, Sakuda M. Open bite diagnosis and treatment. Am J Orthod 1964; 50: 337-358.

28. Mizrahi B. A review of anterior open bite. $\mathrm{Br} \mathrm{J}$ Orthod 1978; 21-27.

29. Kikushi M, Higurashi N, Miyazaki S, Itasaka Y, Chiba S, Nezu H. Facial pattern categories of sleep breathing-disordered children using Ricketts analysis. Psychiatr Clin Neurosci 2002 56(3): 329-330.

30. Juliano ML, Machado MA, Carvalho LC, Lopes EA, Prado GF. Mouth breathing children present a similar cephalometric pattern in OSAS adults patients. Sleep 2004; 26:A134.

31. Proffit WR, Filld HW. Occlusal forces in normal and long face children. J Dent Res 1983; 571-574.

32. Simões WA. Ortopedia Funcional dos Maxilares através da Reabilitação Neuro-oclusal. $3^{a}$ edição. Porto Alegre: Artes Médicas, 2003.

33. Planas P. Reabilitação Neuro-oclusal. $2^{a}$ edição. MassonSalvat, 1994.

\section{Congresso Brasileiro de Neurofisiologia Clínica}

29 de outubro a 02 de novembro de 2005

Gramado - RS

www.vjs.com.br/neuro

\section{Congresso Brasileiro de Sono}

\section{2 a 15 de novembro de 2005}

Curitiba - PR

www.cbsono2005.com.br 